\title{
The Changing Aspects of Motherhood in Face of the COVID-19 Pandemic in Low- and Middle-Income Countries
}

\author{
Jennifer Prince Kingsley ${ }^{1}\left(\mathbb{D} \cdot\right.$ Paul Kingsley Vijay $^{2} \cdot$ Jacob Kumaresan $^{3} \cdot$ Nalini Sathiakumar $^{1}$
}

Accepted: 6 November 2020 / Published online: 26 November 2020

(c) Springer Science+Business Media, LLC, part of Springer Nature 2020

\begin{abstract}
Purpose To advocate perspectives to strengthen existing healthcare systems to prioritize maternal health services amidst and beyond the COVID-19 pandemic in low- and middle income countries.

Description COVID-19 directly affects pregnant women causing more severe disease and adverse pregnancy outcomes. The indirect effects due to the monumental COVID-19 response are much worse, increasing maternal and neonatal mortality.

Assessment Amidst COVID-19, governments must balance effective COVID-19 response measures while continuing delivery of essential health services. Using the World Health Organization's operational guidelines as a base, countries must conduct contextualized analyses to tailor their operations. Evidence based information on different services and comparative cost-benefits will help decisions on trade-offs. Situational analyses identifying extent and reasons for service disruptions and estimates of impacts using modelling techniques will guide prioritization of services. Ensuring adequate supplies, maintaining core interventions, expanding non-physician workforce and deploying telehealth are some adaptive measures to optimize care. Beyond the COVID-19 pandemic, governments must reinvest in maternal and child health by building more resilient maternal health services supported by political commitmentand multisectoral engagement, and with assistance from international partners.

Conclusions Multi-sectoral investments providing high-quality care that ensures continuity and available to all segments of the population are needed. A robust primary healthcare system linked to specialist care and accessible to all segments of the population including marginalized subgroups is of paramount importance. Systematic approaches to digital health care solutions to bridge gaps in service is imperative. Future pandemic preparedness programs must include action plans for resilient maternal health services.
\end{abstract}

Keyword Maternal and child health $\cdot$ Covid $19 \cdot$ Pandemic $\cdot$ Pregnant women $\cdot$ Preparedness

\section{Significance Statement}

\section{What is Already Know on this Subject?}

Jennifer Prince Kingsley

jennypk@uab.edu

Paul Kingsley Vijay

paulkvijay@gmail.com

Jacob Kumaresan

jacobkumaresan@yahoo.com

Nalini Sathiakumar

nalini@uab.edu

1 Department of Epidemiology, University of Alabama at Birmingham, Birmingham, USA

2 Clinic Qualitas, Johor Bahru, Malaysia

3 Former WHO Executive Director, New York, USA
The direct effects of COVID-19 on pregnant women include more severe disease and increased risk of adverse pregnancy outcomes such as preterm births. The indirect effects from barriers to health services are more severe exacerbating maternal/neonatal morbidity and mortality particularly in low- and middle-income countries, with vulnerable subgroups affected the most.

\section{What this Study Adds?}

We advocate perspectives to strengthen existing healthcare systems to prioritize maternal health services amidst and 
beyond the COVID-19 pandemic. Multi-sectoral investments providing high-quality care that ensures continuity and available to all segments of the population are needed.

\section{Introduction}

Since December 2019, the world is reeling from a pandemic of titanic proportions due to Corona Virus Disease 2019 (COVID-19) caused by severe acute respiratory syndrome coronavirus 2 (SARS-CoV-2) (WHO 2020a). As of October 2020, COVID-19 has infected almost 35 million people and has caused more than 1,000,000 deaths globally (JHU 2020). Efforts to stem the COVID-19 pandemic has exposed every country's existing social, economic, geographic and health care access inequalities on an unprecedented scale. The weak health infrastructures coupled with endemic diseases and malnutrition in low- and middle-income countries (LMICs) has further deepened such inequities.

\section{COVID-19 and Pregnancy}

\section{Direct Effects}

The World Health Organization (WHO) classifies pregnant women as an important subgroup of the population that are most vulnerable in the event of a pandemic or a disaster, taking a relatively high share of the disease and associated emergencies (WHO 2002). Viral disease pandemics in the past such as the Severe Acute Respiratory Syndrome (SARS) in 2002-2003 which originated in the Guangdong province of China and the Middle Eastern Respiratory Syndrome (MERS) in 2012 which originated in Saudi Arabia (also caused by coronaviruses) were associated with adverse pregnancy outcomes. SARS was associated spontaneous miscarriages, preterm deliveries, intrauterine growth retardation (IUGR), intensive care hospitalization, and a maternal mortality of 30\% (Maxwell et al. 2017). MERS had similar adverse pregnancy outcomes with a maternal mortality of $27 \%$ and a neonatal mortality of $27 \%$ (Alfaraj et al. 2019). There was no evidence of an intrauterine transmission of either SARS-CoV or MERS-CoV (Schwatz et al. 2020).

Pregnant women are known to be at an increased risk for more severe illness from respiratory infections due to their immunologic and physiologic changes that occur during pregnancy (Rasmussen et al. 2011). Using COVID-19 surveillance data from January to June 2020, the Centers for Disease Control and Prevention (CDC) compared data on pregnant $(\mathrm{n}=8207)$ and nonpregnant $(\mathrm{n}=83,205)$ women who tested positive for SARS-Co-V-2 (Ellington et al. 2020). Compared to nonpregnant women, pregnant women were more likely to be hospitalized ( $31.5 \%$ vs. $5.8 \%$ ), and to be
1.5 times more likely to be admitted to the intensive care unit and to receive mechanical ventilation, but the risk of death was similar ( $0.2 \%$ for both). Data from the CDC COVIDNET on hospitalized pregnant women $(n=598)$ from 13 US states during March to August 2020 with laboratoryconfirmed COVID-19 found that severe illness occurred among symptomatic pregnant women $(\mathrm{n}=272)$ including intensive care admissions (16\%), mechanical ventilation (8\%) and death (1\%) compared to $0 \%$ of these characteristics among asymptomatic pregnant women $(\mathrm{n}=326)$ (Delahoy et al. 2020). Compared to asymptomatic pregnant women, symptomatic pregnant women had higher occurrences of pregnancy loss (5\% vs. $0.9 \%)$ and newborn death $(1.5 \%$ vs. $0 \%)$. A cohort of 1,009 pregnant women going into labor at one of 45 hospitals in Spain during March to May 2020 and who were screened for SARS-Co-V-2 was followed up until six weeks post-partum; 246 tested positive and 763 tested negative (Perez et al. 2020). Compared to the COVID-19 negative women, COVID-19 positive women had a significant two-fold increase in preterm births, 1.7 -fold increase in premature rupture of membranes at term and 4.6-fold increase in neonatal intensive care unit (NICU) admissions. Prematurity and respiratory distress were the main causes of admission to the NICU.

Few other studies, based on small numbers of subjects, provide additional information. Chen et al. (2020) found no evidence of a vertical transmission based on data on six pregnant women with COVID-19 pneumonia in the third trimester. Compared to controls, third trimester placentas in 16 women positive for SARS-CoV-2 were found to show features of maternal vascular mal-perfusion (Shanes et al. 2020). Newborns $(n=10)$ born to mothers with COVID19 exhibited adverse perinatal outcomes such as fetal distress, respiratory distress, thrombocytopenia accompanied by abnormal liver function, and one died (Zhu et al. 2020). Groß et al. (2020) isolated SARS-CoV-2 from one of two nursing mothers with COVID-19. Further longitudinal data from larger study sizes would be required to confirm the findings from the above studies.

The CDC recommends that pregnant women should be aware of their potential risk for severe COVID-19 illness and emphasizes that they take all necessary precautions to avoid exposure to COVID-19; potential barriers to adherence to COVID-19 prevention measures should be addressed (CDC 2020a). Although breast feeding has not been shown conclusively to transmit the virus, the $\mathrm{CDC}$ recommends breast feeding of infants if the mother is asymptomatic in view of the benefits of breast milk.

\section{Indirect Effects}

Governments around the world have responded to the COVID-19 crisis by implementing monumental measures to 
prevent transmission of infection and to treat infected cases. Such response measures have impacted maternal and neonatal health negatively and exacerbated the existing inequalities within societies. Prior to the COVID-19 pandemic, highquality and timely maternal health services were unavailable or inaccessible to large proportions of women in LMICs of very low to low socioeconomic status and those living in rural areas (Bong et al. 2020). The COVID-19 response has further compromised these services.

\section{Disruption of Maternal Health Services}

With the draconian measures required for COVID-19 containment, countries are facing major challenges to maintain the provision and continuity of essential services for pregnant women and newborns. High-income countries (HICs) focused on testing, hospitalization and intensive care, cancelling "non-essential" services such as elective surgeries, restructuring maternity and other wards to treat COVID-19 patients and switching to virtual care when possible (Hart et al. 2020). In LMICs with fewer resources and limited intensive care capacity, governments downscaled or closed regular maternal health services. As per the WHO, essential services have been disrupted, ranging from 24 to $64 \%$ (WHO 2020b). Further, women with high-risk pregnancies have limited access to specially trained providers at secondary or tertiary care facilities, negatively impacting maternal and neonatal morbidity and mortality (Kapur et al. 2015); common causes of high-risk pregnancies include obstructed labor, post-partum hemorrhage, sepsis and maternal noncommunicable diseases (NCDs) such as hyperglycemia in pregnancy, hypertensive disorders of pregnancy, anemia etc. An analysis of data collected from national surveys in 132 LMICs in Africa, Asia, Eastern and Southern Europe, Latin America, and Caribbean reports that an additional 1.7 million pregnant women and 2.6 million newborns would experience major complications and not receive the care they need during the COVID-19 pandemic (Riley et al. 2020).

\section{Diversion of Resources}

Governments have diverted health care resources to COVID-19 response efforts at the expense of several essential services including maternal and child health services; these resources include budgets, personnel, medications, personal protective equipment (PPEs), infection control supplies (disinfectants, sanitizers) etc. In most LMICs, with most health care workers diverted to COVID-19 care, local midwives and community health workers (CHWs) have been the backbone in maintaining routine maternal health services. In India, Accredited Social Health Activists (ASHA) workers instituted by the government's Ministry of Health for the National Rural Health Program continue to provide maternal health services despite lack of supplies such as PPEs (Awasthi, 2020); scarcity of resources pose additional burden on these workers who work in an atmosphere of fear and anxiety of contracting the disease. Several frontline workers and support personnel have been infected with COVID-19 (Nguyen et al. 2020).

\section{Lockdowns/Quarantine}

Most LMICs implemented rigorous strategies to limit the spread of infection via national lockdowns (stay-at-home or movement control order) along with suspension of public transportation. In most LMICs, the poorest families may typically share a single-room dwelling with eight to 10 family members, use a public bathroom and have limited access to public water supply (Madhav et al. 2017). Thus, "social distancing" and hand hygiene practices may not be an option and family members have a high likelihood of contracting and spreading infection, with pregnant women and their newborns being particularly vulnerable. Further, lockdowns/suspended transportation measures have reduced access to care especially among vulnerable groups most in need of care. Further, fear of contracting COVID-19 may cause delays in seeking care. During the Ebola epidemic in West Africa in 2014-2016, pregnant women had fewer or no antenatal care visits and delivered at home for fear of contracting the infection (Sochas et al. 2017). Lack of access to care is particularly life-threatening for high-risk pregnancies and obstetric emergencies. Mental disorders, intimate partner violence (IPV) and poverty are other problems associated with lockdown measures and consequent economic losses. Mental disorders - Worldwide, about $10 \%$ of pregnant women and $13 \%$ of women in the postpartum period develop a mental disorder with LMICs having a higher occurrence of $15.6 \%$ and $19.8 \%$, respectively (WHO 2015a). As depression and anxiety have escalated with the COVID-19 pandemic, the looming mental health crisis is expected to emerge as a major public health problem (Topalidou et al. 2020). IPV - Estimates from the United Nations Population Fund indicate that every three months of quarantine will result in a $20 \%$ increase in IPV throughout the world with at least 15 million additional cases of IPV (Stanley 2020). Social isolation is the major risk factor followed by unemployment and anxiety. IPV victims are at risk of unwanted pregnancies and associated complications (Kaur 2020). Poverty- COVID-19 is pushing almost 70 million people into poverty (Mahler et al. 2020). Poverty is associated with increased risks for preterm labor and delivery, IUGR and neonatal death. The early child health consequences of poverty often sets a newborn on a life-course of health disparities (Larson et al. 2007). 


\section{Maternal and Neonatal Mortality}

During the ongoing COVID-19 crisis, women in LMICs are more likely to deliver at home without a skilled birth attendant leading to deaths from treatable and preventable conditions. In the case of the Ebola epidemic in West Africa, maternal and neonatal deaths and stillbirths indirectly caused by the epidemic outnumbered direct Ebola-related deaths (4000 vs. 3600) (Sochas et al. 2017); indirect causes were primarily due to disruption or shutdown of maternal health services. A similar scenario is observed for the COVID-19 pandemic; the indirect effects of COVID-19 response are of great concern. A study done across in 118 LMICs using the Lives Saved Tool (LiST) estimated that COVID-related maternal health service coverage reduction of $9.8 \%-18.5 \%$ over six months would result in 12,200 additional maternal deaths (Robertson et al. 2020). Coverage reductions of $39.3 \%-51.9 \%$ over 6 months, considered a more serious scenario, would result in 56,700 additional maternal deaths. The additional deaths represent an 8.3 to $38.6 \%$ increase in maternal deaths per month.

Countries with the expected highest numbers of births beginning nine months since the beginning of the COVID-19 pandemic include India (20.1 million), China (13.5 million), Nigeria (6.4 million), Pakistan (5 million) and Indonesia (4 million); these countries had high maternal and neonatal mortality rates prior to the pandemic (Stein, et al, 2020). If essential maternal and neonatal services decline consistently in LMICs, it is estimated that an additional 31,980 maternal deaths, 395,440 newborn deaths and 338,760 stillbirths would occur, corresponding to a $31 \%$ increase in mortality in these indicators.

\section{Perspectives to Maintain and Strengthen Maternal Health Services}

\section{Amidst the COVID-19 Pandemic}

Maternal and neonatal mortality rates are key indicators of the health and infrastructure of a country and governments across the world have strived to improve the health of pregnant women and newborns. In 2015, the United Nations (UN) reviewed the Millennium Development Goal (MDG) 5 to reduce global maternal mortality ratio (MMR) by $75 \%$ and found that only few countries had achieved this target. In the same year, the UN Summit for Sustainable Development, adopted 17 Sustainable Development Goals (SDGs) for 2030: SDG 3 targets reducing the global MMR to no more than 70 maternal deaths/100,000 live births, with a supplementary national target that no country should have an MMR greater than 140/100,000 live births, and reducing the neonatal mortality rate (NMR) to no more than 12 infant deaths/1000 live births (WHO 2015b). The global progress towards these targets is lagging. Failure to prioritize maternal health during the COVID-19 pandemic would reverse the gains made in MMR and NMR during the past decade. The impact would be enormous for LMICs and marginalized populations within them.

Policymakers in LMICs face the major dilemma of balancing effective COVID-19 response measures with continued delivery of essential health services because of their low-resilience health systems. These efforts will require sufficient financial and technical resources. International funding partners have stepped up to provide some support, necessitating LMICs to maximize their resources. In implementing action plans, the WHO's operational planning guidelines for maintaining essential services at national, regional and local levels during the COVID-19 pandemic will form the base (WHO 2020c). As resources and disease burdens differ by country, decision makers in each country must conduct contextualized analysis to tailor operations to prioritize eessential services with trade-offs on strategy of other services of a lesser priority to ensure that limited resources provide maximum benefit for the population. Evidence-based information on different services and comparative cost-benefits will guide these decisions; global development partners play a major role in providing technical assistance in this process.

\section{Identifying Extent and Reasons for Service Disruptions and Impacts}

An initial step would be a situational analysis with quantitative and qualitative components conducted among stakeholders including local experts and community members. The extent and reasons for service disruptions in the continuum of maternal health services from antenatal care, delivery and postnatal/neonatal care must be ascertained. Projection modelling tools are valuable in providing quantitative estimates of impacts of service disruptions on maternal and neonatal morbidity and mortality. In evaluating the impact of disruption of specific services, comparative analysis of COVIDspecific health benefits may be weighed against the greater health benefits of continuing such services. Health impacts on frontline health workers, who are critical resources in the health care system, will provide the basis to ensure adequate resources to minimize their exposures. Stratifying all analyses by population subgroups will help in identifying vulnerable groups for the purpose of ensuring inclusive services that reduce inequities.

\section{Adaptation of Strategies to Optimize Services}

National governance should prioritize maternal and neonatal services, issue specific and practical guidance on 
the maintenance of services and monitor the continuity and access to services. Prioritizing maternal and neonatal services as "essential" will enable all pregnant women including those with suspected or confirmed COVID-19 to continue antenatal care visits, deliver in a functional facility with a skilled birth attendant and have access to post-natal and neonatal care. Health care systems must be well-equipped to provide emergency antenatal, natal and postnatal care. It is crucial to include targeted plans for vulnerable subgroups of the population who are most at high risk for both the direct and the indirect effects of COVID-19. Some approaches include: (1) Provision of adequate supplies including PPEs and disinfectants, and ensuring continuity of essential medications. (2) Maintaining core maternal and child interventions that are known to prevent deaths. Some examples are: parenteral administration of uterotonics, antibiotics, anticonvulsants and clean birth environments prevent $60 \%$ of additional maternal deaths; coverage of antibiotics for neonatal sepsis and pneumonia and oral rehydration for diarrhea prevent $41 \%$ of additional neonatal deaths (Robertson et al. 2020). (3) Mobilizing CHWs to provide education, triaging and referrals for COVID-19 supported with sufficient funding, technical support and more personnel. (4) COVID-dedicated clinics and wards for the care of pregnant women who are COVID-19 positive with separate entrances and restricted access to visitors (CDC 2020b). (5) Deploying telehealth and other digital tools. In LMICs, cell phone penetration is about $90 \%$ in recent years and mobile internet connectivity is around 40\% (Feroz et al. 2020). LMICs have used mHealth extensively for education and prevention measures for COVID-19. For vulnerable subgroups with no access to cell phones, alternative strategies such as campaigns should be considered. Use of mobile phone-based telehealth measures to replace in-person consults provide scope for innovation and experimentation for maintaining continuity of services and referrals. (6) Mental health services should be a part of the agenda with stepping up of services to tackle the increased demands and integrated into primary healthcare. (7) Transparent communications with the public and strong community engagement to maintain people's trust in the system to facilitate their seeking care when appropriate, and adhering to public health advice.

\section{Beyond the COVID-19 Pandemic}

Beyond COVID-19, LMIC governments will look at new ways to develop innovative programs and strategies to reduce the vast inequalities and build more resilient and inclusive societies. It is clear that a focus on maternal and child health will promote later resilience. The COVID-19 pandemic offers a unique opportunity for LMICs to reinvest in maternal and child health in the post-COVID-19 period. Implementing appropriate policies will be challenging and will require political commitment and multisectoral engagement. In addressing the historic underinvestment in maternal and child health, LMICs must build new partnerships and expand international funding partners. Investments that will generate the greatest benefit should be prioritized based on evidence-based data. The consequences of poor maternal and child health with adverse trajectories extending to adulthood and to the next generation (eg. maternal malnutrition leads to childhood stunting and low educational attainment in adulthood, low birthweight predisposing to NCDs in adult life etc.) must factor in these decisions. A robust primary healthcare system linked to specialist care and accessible to all segments of the population including marginalized subgroups is paramount to optimizing maternal and neonatal health. Developing systematic approaches to digital health care solutions to bridge gaps in service is imperative. Action plans for resilient maternal health services should be integral in future pandemic preparedness programs.

\section{Conclusions}

As aptly summed up by Henrietta Fore, UNICEF Executive Director, "Millions of mothers all over the world embarked on a journey of parenthood in the world as it was. They now must prepare to bring a life into the world as it has become-a world where expecting mothers are afraid to go to health centers for fear of getting infected, or missing out on emergency care due to strained health services and lockdowns. It is hard to imagine how much the coronavirus pandemic has recast motherhood" (Africa News 2020). While it is necessary to focus on the current crisis, maternal health must be protected through research, advocacy and practice. The pandemic gives us the unique opportunity to reassess and address the disparities in our society. Being vigilant and sensitive to the changing needs is crucial to maintain continuity of care for pregnant women and to provide for the next generation coming into the world in such tumultuous times.

Authors Contribution JPK designed the review and drafted the article. PKV contributed to the revision and re-drafting of the article. JK and NS reviewed it for intellectual content and approved the version to be published.

Funding None.

\section{Compliance with Ethical Standards}

Conflict of interest The Authors declare no conflict-of-interests. 


\section{References}

Africa News. Coronavirus - Africa: Pregnant mothers and babies born during COVID-19 pandemic threatened by strained health systems and disruptions in services. Africa News. https://www.africanews .com/2020/05/07/coronavirus-africa-pregnant-mothers-and-babie s-born-during-covid-19-pandemic-threatened-by-strained-healt h-systems-and-disruptions-in-services//

Alfaraj, S. H., Al-Tawfiq, J. A., \& Memish, Z. A. (2019). Middle East respiratory syndrome coronavirus (MERS-CoV) infection during pregnancy: Report of two cases \& review of literature. Journal of Microbiology Immunology and Infection, 52, 501-503.

Awasthi P., The life of ASHA workers in the time of COVID-19. For ASHA workers, the pandemic has meant more hours of work and personal risk. The Week. https://www.theweek.in/news/india /2020/04/10/the-life-of-asha-workers-in-the-time-of-covid-19. html. Accessed 10 April 2020.

Bong C. L., Brasher C., Chikumba E., McDougall R., Mellin-Olsen J., Enright A. (2020). The COVID-19 Pandemic: Effects on Lowand Middle-Income Countries. Anesthesia \& Analgesia.;131(1), 86-92. https://doi.org/https://doi.org/10.1213/ANE.0000000000 004846. https://pubmed.ncbi.nlm.nih.gov/32243287/

Centers for Disease Control and Prevention (CDC, 2020a). 9 June, 2020. If You Are Pregnant, Breastfeeding, or Caring for Young Children. https://www.cdc.gov/coronavirus/2019-ncov/need-extra -precautions/pregnancy-breastfeeding.html.

Centers for Disease Control and Prevention (CDC, 2020b) 15 September, 2020. Healthcare Workers; Management of Visitors to Healthcare Facilities in the Context of COVID-19: Non-US Healthcare Settings https://www.cdc.gov/coronavirus/2019-ncov/hcp/non-ussettings/hef-visitors.html.

Chen, H., et al. (2020). Clinical characteristics and intrauterine vertical transmission potential of COVID-19 infection in nine pregnant women: aretrospective review of medical records. Lancet, 395(10226), 809-815. https://doi.org/10.1016/S0140 -6736(20)30360-3.

Delahoy, M. J., Whitaker, M., O'Halloran, A., et al (2020). Characteristics and Maternal and Birth Outcomes of Hospitalized Pregnant Women with Laboratory Confirmed COVID 19 - COVID-NET, 13 States, March 1-August 22,2020. MMWR Morb Mortal Wkly Rep. https://doi.org/https://doi.org/10.15585/mmwr.mm6938e1

Ellington, S., Strid, P., Tong V. T., et al. (2020). Characteristics of Women of Reproductive Age with Laboratory-confirmed SARSCoV-2 Infection by Pregnancy Status - United States, January 22 - June 7, 2020. MMWR Morbidity and Mortality Weekly Report, 769-775. https://doi.org/https://doi.org/10.15585/mmwr.mm692 $5 \mathrm{a} 1$

Feroz, A., Jabeen, R., \& Saleem, S. (2020). Using mobile phones to improve community health workers performance in low-andmiddle-income countries. BMC Public Health, 20(1), 49. https:// doi.org/10.1186/s12889-020-8173-3.

Groß, R., Conzelmann, C., Müller, J. A., et al. (2020). Detection of SARS-CoV-2 in human breastmilk. Lancet, 395(10239), 17571758. https://doi.org/10.1016/S0140-6736(20)31181-8.

Hart, A., Teegardin, C. (2020) The Atlanta Journal-Constitution, Preparing for surge, hospitals limit visitors, reschedule surgeries. https://www.ajc.com/news/state--regional/preparing-for-surge -hospitals-limit-visitors-reschedule-surgeries/OqnPmLZhmM hwCu5jQ6J6oBK/. Accessed 1 April 2020.

Johns Hopkins University of Medicine (JHU), Coronavirus Resource Center. https://coronavirus.jhu.edu. Accessed 21 June 2020.

Kapur, A. (2015). Links between maternal health and NCDs. Best Practice \& Research: Clinical Obstetrics \& Gynaecology, 29(1), 32-42. https://doi.org/https://doi.org/10.1016/j.bpobg yn.2014.04.016. https://pubmed.ncbi.nlm.nih.gov/25199858/
Kaur, J. (2020). COVID-19 lockdowns leading to a rise in violence against women and girls. International Federation of Obstetrics and Gynecology,. https://www.figo.org/covid-19-lockdowns-leadi ng-rise-violence-against-women-and-girls.

Larson, C. P. (2007). Poverty during pregnancy: Its effects on child health outcomes. Paediatrics \& Child Health, 12(8), 673-677. https://doi.org/https://doi.org/10.1093/pch/12.8.673. https://acade mic.oup.com/pch/article/12/8/673/2647987.

Madhav, N., Oppenheim, B., Gallivan, M., et al. (2017). Pandemics: Risks, Impacts, and Mitigation. In Jamison D. T., Gelband H., Horton S., et al. (Ed). Disease Control Priorities: Improving Health and Reducing Poverty. 3rd edition. Washington (DC): The International Bank for Reconstruction and Development/The World Bank, Chapter 17. https://doi.org/https://doi. org/10.1596/978-1-4648-0527-1_ch17. https://www.ncbi.nlm. nih.gov/books/NBK525302/.

Mahler, D. G., Lakner, C., Aguilar, A. C., Wu, H. (2020). Updated estimates of the impact of COVID-19 on global poverty. World Bankorg. https://blogs.worldbank.org/opendata/updated-estim ates-impact-covid-19-global-poverty.

Maxwell, C., McGeer, A., Tai, K. F. Y., \& Sermer, M. (2017). Management guidelines for obstetric patients and neonates born to mothers with suspected or probable severe acute respiratory syndrome (SARS). Journal of Obstetrics and Gynaecology Canada, 39, e130-e137. https://doi.org/10.1016/j.jogc.2017.04.024.

Nguyen, L. H., et al. (2020). Risk of COVID-19 among front-line health-care workers and the general community: A prospective cohort study. The Lancet Public Health, 5(9), e475-e483. https:// doi.org/10.1016/S2468-2667(20)30164-X.

Perez, O. M., et al. (2020). The association between COVID-19 and preterm delivery: A cohort study with a multivariate analysis. medRxiv: 2020.2009.2005.20188458. https://www.medrxiv.org/ content/medrxiv/early/2020/09/07/2020.09.05.20188458.full.pdf

Rasmussen, S. A., Kissin, D. M., Yeung, L. F., MacFarlane, K., Chu, S. Y., Turcios-Ruiz, R. M., et al. (2011). Preparing for influenza after 2009 H1N1: Special considerations for pregnant women and newborns. American Journal of Obstetrics and Gynecology, 204(6 Suppl 1), S13-S20. https://doi.org/10.1016/j.ajog.2011.01.048.

Riley, T., Sully, E., Ahmed, Z., Biddlecom, A. (2020). Estimates of the Potential Impact of the COVID-19 Pandemic on Sexual and Reproductive Health In Low- and Middle-Income Countries. International Perspectives on Sexual and Reproductive Health, 46, 73-76. https://doi.org/https://doi.org/10.1363/46e90 20. https://www.guttmacher.org/journals/ipsrh/2020/04/estim ates-potential-impact-covid-19-pandemic-sexual-and-reproducti ve-health.

Robertson, T., Carter, E. D., Chou, V. B., et al. (2020). Early estimates of the indirect effects of the COVID-19 pandemic on maternal and child mortality in low-income and middle-income countries: a modelling study. The Lancet Global Health, 8(7), E901-E908. https://doi.org/10.1016/S2214-109X(20)30229-1.

Schwartz, D. A. (2020). An Analysis of 38 Pregnant Women with COVID-19, Their Newborn Infants, and Maternal-Fetal Transmission of SARS-CoV-2: Maternal Coronavirus Infections and Pregnancy Outcomes. Archives of Pathology \& Laboratory Medicine.https://doi.org/https://doi.org/10.5858/arpa.2020-0901-SA. https://www.ncbi.nlm.nih.gov/pubmed/32180426. Accessed 12 June 2020.

Shanes, E. D., Mithal, L. B., Otero, S., Azad, H. A., Miller, E. S., \& Goldstein, J. A. (2020). Placental pathology in COVID-19. American Journal of Clinical Pathology, 154(1), 23-32. https:// doi.org/10.1093/ajcp/aqaa089.

Sochas, L., Channon, A. A., Nam, S. (2017). Counting indirect crisisrelated deaths in the context of a low-resilience health system: the case of maternal and neonatal health during the Ebola epidemic in Sierra Leone. Health Policy Plan, 32(3), iii32-iii39. https://doi. 
org/https://doi.org/10.1093/heapol/czx108. https://pubmed.ncbi. nlm.nih.gov/29149310/.

Stanley, M. J. D. (2020). Why the Increase in Domestic Violence During COVID-19? COVID-19 has triggered common factors associated with domestic violence. Psychology Today, https://www. psychologytoday.com/us/blog/making-sense-chaos/202005/whythe-increase-in-domestic-violence-during-covid-19.

Stein, D., Ward, K., Cantelmo, C. (2020). Estimating the Potential Impact of COVID-19 on Mothers and Newborns in Low- and Middle-Income Countries. Health Policy Pluscom. http://www. healthpolicyplus.com/covid-mnh-impacts.cfm.

Topalidou, A., Thomson, G., Downe, S. (2020). COVID-19 and maternal mental health: Are we getting the balance right? UK. medRxiv preprint. https://doi.org/https://doi.org/10.1101/2020.03.30.20047 969. https://www.medrxiv.org/content/https://doi. org/10.1101/2020.03.30.20047969v1.full.pdf.

World Health Organization (WHO, 2002) Environmental health Emergencies, Vulnerable groups. https://www.who.int/environmen tal_health_emergencies/vulnerable_groups/en/

World Health Organization, WHO International (2015a), Maternal Mental health. https://www.who.int/mental_health/maternal-child /maternal_mental_health/en/. Accessed 20 Feb 2015.

World Health Organization (WHO, 2015b). Trends in Maternal Mortality: 1990-2015 -Estimates by WHO, UNICEF, UNFPA, World Bank Group, and the United Nations Population Fund.
2015. http://apps.who.int/iris/bitstream/10665/194254/1/9 789241565141_eng.pdf?ua $=1$.

World Health Organization (WHO, 2020a) Novel Coronavirus - China. Disease outbreak news: Update. https://www.who.int/csr/don/12january-2020-novel-coronavirus-china. Accessed 12 Jan 2020.

World Health Organization (WHO, 2020b), Rapid assessment of service delivery for NCDs during the COVID-19 pandemic. https ://www.who.int/publications/m/item/rapid-assessment-of-servi ce-delivery-for-ncds-during-the-covid-19-pandemic. Accessed 29 May 2020.

World Health Organization (WHO, 2020c); 1 June 2020. Maintaining essential health services: operational guidance for the COVID19 context interim guidance. https://www.who.int/publications/i/ item/WHO-2019-nCoV-essential-health-services-2020.1.

Zhu, H., Wang, L., Fang, C., Peng, S., Zhang, L., Chang, G., Xia, S., \& Zhou, W. (2020). Clinical analysis of 10 neonates born to mothers with 2019-nCoV pneumonia. Translational pediatrics, 9(1), 51-60. https://doi.org/https://doi.org/10.21037/tp.2020.02.06

Publisher's Note Springer Nature remains neutral with regard to jurisdictional claims in published maps and institutional affiliations. 